Received: 8 August 2017

Accepted: 22 November 2017

Published online: 17 January 2018

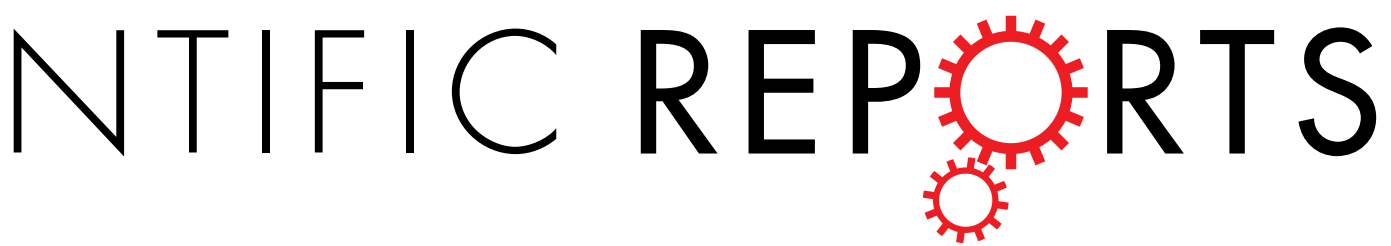

\title{
Increased left ventricular mass index is present in patients with type 2 diabetes without ischemic heart disease
}

Jelena P. Seferovic $c^{1,6}$, Milorad Tesic ${ }^{2,6}$, Petar M. Seferovic ${ }^{2,6}$, Katarina Lalic ${ }^{1,6}$, Aleksandra Jotic ${ }^{1,6}$, Tor Biering-Sørensen ${ }^{3}$, Vojislav Giga ${ }^{2,6}$, Sanja Stankovic ${ }^{4}$, Natasa Milic ${ }^{5}$, Ljiljana Lukic ${ }^{1,6}$, Tanja Milicic ${ }^{1,6}$, Marija Macesic ${ }^{1,6}$, Jelena Stanarcic Gajovic ${ }^{1}$ \& Nebojsa M. Lalic ${ }^{1,6}$

Left ventricular mass index (LVMI) increase has been described in hypertension (HTN), but less is known about its association with type 2 diabetes (T2DM). As these conditions frequently co-exist, we investigated the association of T2DM, HTN and both with echocardiographic parameters, and hypothesized that patients with both had highest LVMI, followed by patients with only T2DM or HTN. Study population included 101 T2DM patients, 62 patients with HTN and no T2DM, and 76 patients with T2DM and HTN, excluded for ischemic heart disease. Demographic and clinical data, biochemical measurements, stress echocardiography, transthoracic 2D Doppler and tissue Doppler echocardiography were performed. Multivariable logistic regression was used to determine the independent association with T2DM. Linear regression models and Pearson's correlation were used to assess the correlations between LVMI and other parameters. Patients with only T2DM had significantly greater LVMI $\left(84.9 \pm 20.3 \mathrm{~g} / \mathrm{m}^{2}\right)$ compared to patients with T2DM and HTN $\left(77.9 \pm 16 \mathrm{~g} / \mathrm{m}^{2}\right)$ and only HTN $\left(69.8 \pm 12.4 \mathrm{~g} / \mathrm{m}^{2}\right)$. In multivariate logistic regression analysis, T2DM was associated with LVMI (OR 1.033, 95\% $\mathrm{Cl} 1.003-1.065, \mathrm{p}=0.029$ ). A positive correlation of $\mathrm{LVMI}$ was found with fasting glucose $(p<0.001)$ and HbAlc $(p=0.0003)$. Increased LVMI could be a potential, pre-symptomatic marker of myocardial structural change in T2DM.

Cardiovascular diseases (CV) are the leading cause of morbidity and mortality in patients with type 2 diabetes mellitus (T2DM) ${ }^{1}$. Also, T2DM is considered a major co-morbidity and a strong independent risk factor for the development and progression of heart failure $(\mathrm{HF})^{2}$, with both preserved or reduced ejection fraction $(\mathrm{EF})^{3}$. It has been shown that worse glycemic control has been associated with worsening of cardiac structure and function $^{4}$.

There is evidence suggesting that T2DM could be associated with increased left ventricular (LV) mass ${ }^{5,6}$, concentric geometry/remodeling, and impaired diastolic function ${ }^{7}$. Over time, these structural and functional changes result in impaired systolic function and symptomatic HF, which are associated with worse clinical outcomes $^{8}$. Hypertension (HTN) has previously been associated with elevated LV mass ${ }^{9}$. Also, it is well known that LV mass increases with age, obesity, dyslipidemia, which are often present in T2DM and HTN ${ }^{10,11}$. Therefore, these patients have a higher incidence of increased LV mass, as well as other myocardial impairments, in comparison to patients without multiple co-morbidities ${ }^{12-14}$

${ }^{1}$ Clinic of Endocrinology, Diabetes and Metabolic disorders, Clinical Center of Serbia, Dr. Subotica 13, 11000, Belgrade, Serbia. ${ }^{2}$ Clinic of Cardiology, Clinical Center of Serbia, Koste Todorovica 8, 11000, Belgrade, Serbia. ${ }^{3}$ Department of Cardiology, Herlev and Gentofte Hospital, University of Copenhagen, Copenhagen, Denmark. ${ }^{4}$ Center for Medical Biochemistry, Clinical Center of Serbia, Visegradska 26, 11000, Belgrade, Serbia. ${ }^{5}$ Institute of Medical Statistics, Faculty of Medicine, Dr. Subotića 15, Belgrade, Serbia. ${ }^{6}$ Present address: University of Belgrade, Faculty of Medicine, Belgrade, Serbia. Correspondence and requests for materials should be addressed to J.P.S. (email: jpseferovic@gmail.com) 
We therefore investigated the association of T2DM, HTN and both co-morbidities with LV mass measured by echocardiography. We hypothesized that patients with both T2DM and HTN had highest LV mass, followed by patients with only T2DM or HTN.

\section{Methods}

The study population included 101 T2DM patients with normal blood pressure (BP), 62 patients with HTN and no T2DM, and 76 patients with T2DM and HTN. Ischemic heart disease was excluded in all patients. None had prior history or symptoms of heart failure (chest pain, dyspnea, arrhythmia and synkopee), cerebrovascular or renal disease, microvascular diabetic complications or insulin therapy. T2DM was diagnosed based on laboratory data (glycated hemoglobin (HbAlc) $\geq 6.5 \%$ ), medical history of T2DM or therapy (sulfonylurea and/ or metformin). HTN was diagnosed using BP levels higher than $140 / 90 \mathrm{mmHg}$ or antihypertensive treatment (renin-angiotensin system inhibitors, angiotensin II-receptor antagonist, calcium channel blockers, diuretics and beta-blockers, alone or in combination) ${ }^{15}$. Patients were prospectively recruited from the Clinic of Endocrinology, Diabetes and Metabolic Disorders and Clinic of Cardiology, Clinical Center of Serbia, between October 2007 and May 2013.

Demographic and clinical data, as well as anthropometric, echocardiographic and biochemical measurements were obtained for all patients. The study protocol was in adherence to the contents of the Declaration of Helsinki. Informed consent was obtained for all participants and the Medical Ethical Committee of the Clinical Center of Serbia approved the study protocol.

Blood samples were collected following 12-hour fasting [serum glucose, HbAlc, lipid parameters, creatinine], and were analyzed using standard methods. Insulin was assessed by radioimmunoassay method using a commercially available kit. Kidney function was evaluated using the Modification of Diet in Renal Disease Study equation (MDRD) for estimating glomerular filtration rate with standardized serum creatinine ${ }^{16}$. Albumin/creatinine ratio was done in a first morning urine sample. Insulin sensitivity was estimated by Homeostasis Model Assessment of Insulin Resistance (HOMA-IR) ${ }^{17}$, using the formula HOMA-IR = insulin $(\mathrm{mU} / \mathrm{l}) \times$ glycaemia $(\mathrm{mmol} / \mathrm{l}) / 22.5$. Antihiperglycemic therapy was discontinued 48-72 hours prior to blood sampling. Body mass index (BMI) was calculated using the following formula weight $(\mathrm{kg}) / \mathrm{height}(\mathrm{m})^{2}$. BP was measured in supine position with a cuff adjusted to arm circumferential after at least 5 minutes of rest. Stress echocardiography test was used to exclude ischemic heart diseases (Del Mar and Agilent Image Point, USA) using the Bruce protocol ${ }^{18}$. Myocardial ischemia was defined as occurrence of new wall motion abnormality (hypokinesia/akinesia). All patients underwent transthoracic 2D Doppler and tissue Doppler echocardiography using Sequoia c256 Acuson (Siemens Mountain View, California, USA). Standard two dimensional, M-Mode, pulsed Doppler measures were done according to the updated Recommendations for cardiac chamber quantification by echocardiography in adults ${ }^{19}$. Atrial volumes and ejection fraction (EF) were assessed using the modified Simpson biplane method ${ }^{19}$. LV mass was calculated using the Devereux formula and normalized by body surface area (LV mass index [LVMI] $)^{20}$. Relative wall thickness (RWT) was calculated as 2 times PW divided by the LV diastolic diameter ${ }^{21}$. Early and late diastolic peak filling velocities E and A wave were measured at the mitral leaflet tips. The early (e') and late (a') diastolic velocities at septal and lateral corner of mitral annulus were assessed with pulse-wave Tissue Doppler from a standard apical 4 -chamber view ${ }^{19}$. Filters were set to exclude high frequency signals, while direction of annulus motion was aligned with the scan line direction. An experienced investigator, blinded for the clinical data, performed and interpreted all echo-Doppler recordings.

Statistical analysis. Baseline characteristics, laboratory analysis and echocardiographic data were stratified by the presence of T2DM, HTN or both. Descriptive data is presented as the mean \pm standard deviation for normally distributed variables and as median [25-75th percentile] for non-normally distributed variables. Categorical variables are expressed as absolute numbers with percentages and were compared by the $\chi^{2}$-test, while continuous variables were compared using univariate one-way ANOVA or Kruskal-Wallis analysis of variance, as appropriate. Multivariable logistic regression model including all significantly different baseline characteristics and echocardiographic parameters was used to determine the independent association with T2DM. Linear regression models and Pearson's correlation were used to assess the correlations between LVMI and other parameters. Two-sided P-values $<0.05$ were considered significant. Analyses were performed using SPSS Windows version 21.0.

Data availability. The datasets used and/or analyzed during the current study available from the corresponding author on reasonable request.

\section{Results}

The study population included 239 patients (51.5\% males, mean age $55.4 \pm 8.5$ years): 101 T2DM and no HTN, $62 \mathrm{HTN}$ and no T2DM, and 76 patients with both T2DM and HTN. All patients were excluded for ischemic heart disease. Patient characteristics and baseline laboratory data are presented in Table 1. Patients who had only T2DM were predominantly male, and $48 \%$ had a family history of T2DM. Also, they had higher fasting glucose, insulin, HOMA-IR and used metformin and statin more frequently. Although both were within normal ranges, creatinine was significantly higher, while eGFR was lower in these, compared to other patients. Patients who had both T2DM and HTN were older, with a significantly longer duration of T2DM, higher BMI, HbA1c and triglycerides.

Echocardiographic parameters are presented in Table 2. Although systolic function was normal in all patients, EF was significantly lower, while left ventricular end systolic and end diastolic diameter, as well as stroke volume, were significantly higher in patients with only T2DM. LV mass and LVMI were highest in patients with T2DM, followed by patients with both co-morbidities and were lowest in patients with only HTN. Deceleration time was 


\begin{tabular}{|c|c|c|c|c|c|}
\hline Parameter & $\begin{array}{l}\text { T2DM, no HTN* } \\
\mathrm{n}=101\end{array}$ & $\begin{array}{l}\text { HTN, no T2DM** } \\
\mathrm{n}=62\end{array}$ & $\begin{array}{l}\text { T2DM and HTN } * * * \\
\mathbf{n}=76\end{array}$ & $\mathbf{p}$ & $\begin{array}{l}\text { Post hoc multiple } \\
\text { comparisons }\end{array}$ \\
\hline Age, years & $54.5 \pm 9.0$ & $54.3 \pm 8.0$ & $57.4 \pm 7.8$ & 0.04 & \\
\hline Male sex, n (\%) & $64(63)$ & $21(34)$ & $38(50)$ & 0.001 & *vs** \\
\hline \multicolumn{6}{|l|}{ Duration of disease, years } \\
\hline T2DM & $4.0(2.0-5.0)$ & & $5.0(3.0-10.0)$ & $<0.001$ & \\
\hline HTN & & $5.0(2.0-8.0)$ & $5.0(2.0-8.0)$ & 0.23 & \\
\hline \multicolumn{6}{|l|}{ Family history, n (\%) } \\
\hline T2DM & $48(48)$ & $16(26)$ & $33(43)$ & 0.02 & $* \mathrm{vs} * *$ \\
\hline CVD & $40(40)$ & $49(79)$ & $48(63)$ & $<0.001$ & $* \mathrm{vs} * * ; \mathrm{vs}^{* * *}$ \\
\hline Smoking, n (\%) & $37(37)$ & $27(44)$ & $18(24)$ & 0.04 & $* * \mathrm{vs} * * * ; * \mathrm{vs} * * *$ \\
\hline Body mass index, $\mathrm{kg} / \mathrm{m}^{2}$ & $26.7(24.7-29.5)$ & $25.6(23.7-27.7)$ & $28.9(26.0-30.9)$ & $<0.001$ & $* * \mathrm{vs} * * *$ \\
\hline \multicolumn{6}{|l|}{ Blood pressure, $\mathrm{mmHg}$} \\
\hline Systolic & $130(120-130)$ & $150(145-150)$ & $150(145-150)$ & $<0.001$ & *vs**; *vs*** \\
\hline Diastolic & $80(75-80)$ & $95(95-95)$ & $95(95-100)$ & $<0.001$ & $* \mathrm{vs}^{* *} ; * \mathrm{vs} * * *$ \\
\hline \multicolumn{6}{|l|}{ Medications, n (\%) } \\
\hline Metformin & $97(96)$ & & $67(88)$ & 0.05 & \\
\hline Sulfonylurea & $52(52)$ & & $47(62)$ & 0.17 & \\
\hline Statin & $24(24)$ & $11(18)$ & $7(9)$ & 0.04 & $* \mathrm{vs} * * *$ \\
\hline ACE inhibitor & & $42(68)$ & $60(79)$ & 0.14 & \\
\hline $\begin{array}{l}\text { Angiotensin II receptor } \\
\text { blocker }\end{array}$ & & $4(7)$ & $4(5)$ & 0.77 & \\
\hline Diuretic & & $20(32)$ & $11(15)$ & 0.01 & \\
\hline Calcium antagonist & & $25(40)$ & $32(42)$ & 0.83 & \\
\hline Beta blocker & & $31(50)$ & $33(43)$ & 0.44 & \\
\hline Fasting glucose, mmol/l & $7.9(6.7-9.1)$ & $5.5(5.0-5.9)$ & $7.8(6.3-9.0)$ & $<0.001$ & $* \mathrm{vs} * * ; * * \mathrm{vs} * * *$ \\
\hline Glycated hemoglobin, \% & $7.2 \pm 1.0$ & $5.6 \pm 0.6$ & $7.4 \pm 1.1$ & $<0.001$ & $* \mathrm{vs} * * ; * * \mathrm{vs} * * *$ \\
\hline Insulin, $\mathrm{mU} / \mathrm{L}$ & $16.7(11.5-24.6)$ & $10.4(7.0-15.9)$ & $11.9(7.3-16.6)$ & $<0.001$ & $* \mathrm{vs} * * ; * \mathrm{vs} * * *$ \\
\hline HOMA-IR & $5.3(4.0-8.4)$ & $2.5(1.7-4)$ & $3.7(2.3-5.6)$ & $<0.001$ & *vs**; *vs*** \\
\hline Total cholesterol, mmol/l & $5.7 \pm 1.3$ & $6.2 \pm 1.1$ & $5.8 \pm 1.2$ & 0.03 & $* \mathrm{vs}^{* *}$ \\
\hline LDL cholesterol, mmol/l & $3.6 \pm 1.1$ & $4.1 \pm 0.9$ & $3.4 \pm 1.2$ & 0.002 & $* \mathrm{vs} * * ; * * \mathrm{vs} * * *$ \\
\hline HDL cholesterol, mmol/l & $1.1 \pm 0.3$ & $1.4 \pm 0.3$ & $1.2 \pm 0.3$ & $<0.001$ & $* \mathrm{vs} * * ; * * \mathrm{vs} * * *$ \\
\hline Triglycerides, mmol/l & $1.9(1.4-2.7)$ & $1.4(1.1-1.8)$ & $1.9(1.5-2.8)$ & $<0.001$ & $* \mathrm{vs}^{* *} ; * * \mathrm{vs} * * *$ \\
\hline Creatinine, $\mu \mathrm{mol} / \mathrm{l}$ & $83.1 \pm 15.4$ & $69.7 \pm 12.1$ & $70.5 \pm 15$ & $<0.001$ & $* \mathrm{vs} * * ; * \mathrm{vs} * * *$ \\
\hline $\mathrm{eGFR}, \mathrm{ml} / \mathrm{min} / 1.73 \mathrm{~m}^{2}$ & $83 \pm 18.7$ & $92 \pm 14.5$ & $95.5 \pm 20.2$ & $<0.001$ & $* \mathrm{vs} * * ; * \mathrm{vs} * * *$ \\
\hline
\end{tabular}

Table 1. Baseline characeristics and laboratory data. ANOVA; Data is presented as means $\pm \mathrm{SD}$, median [25-75th percentile], or percentages; T2DM-type 2 diabetes, HTN-hypertension, CVD-cardiovascular diseases; HOMA-IR-homeostatic model assessment of insulin resistance index, HDL-high density lipoprotein, LDL-low density lipoprotein, eGFR-estimated glomerular filtration rate.

shortest in T2DM patients, followed by the HTN group and patients with both co-morbidities. The differences in echocardiographic parameters among groups remained significant when evaluated by gender (Supplemental Table 1).

In multivariable logistic regression analysis, T2DM was associated with $\mathrm{BMI}(\mathrm{OR}=1.200,95 \% \mathrm{CI} 1.070-1.347$, $\mathrm{p}=0.002)$, diastolic BP $(\mathrm{OR}=0.847,95 \% \mathrm{CI} 0.768-0.934, \mathrm{p}=0.001)$, and LVMI (OR 1.033 95\%CI 1.003-1.065, $\mathrm{p}=0.029$; Table 3). In multivariable linear regression analysis LVMI was associated with T2DM (standarized $\beta=0.143, p=0.037$ ), male sex (standarized $\beta=-0.254, \mathrm{p}<0.001$ ), mean $\mathrm{E} / \mathrm{E}$ ' ratio (standarized $\beta=0.268$, $\mathrm{p}<0.001$ ), hypertension (standarized $\beta=-0.179, \mathrm{p}=0.009$ ) and $\mathrm{E} / \mathrm{A}$ ratio (standarized $\beta=-0.143, \mathrm{p}=0.016$; Table 4). Also, a positive correlation of LVMI was found with fasting glucose, HbA1c, and creatinine. In addition, LVMI correlated with male sex, HDL cholesterol, systolic and diastolic blood pressures, and eGFR (Table 5, Figs 1 and 2).

\section{Discussion}

In our study, patients with T2DM and no HTN, free of ischemic heart disease, had significantly larger LVMI in comparison to patients with both T2DM and HTN, and those with only HTN. In multivariable analysis, T2DM was significantly associated with LVMI. In addition, increasing LVMI was positively associated with fasting glucose and $\mathrm{HbAlc}$.

In the real clinical setting, patients with T2DM usually have co-existing HTN, both known to contribute to the increase of LVMI. Therefore, we expected to find the highest LVMI in that group, which was not the case. HTN per se is a well-known cause of LVM increase ${ }^{8,22-24}$. However, in our study, investigated patients with only HTN had smallest LVMI. Our results are in contrast to the Strong Heart Study, which showed that the combination 


\begin{tabular}{|c|c|c|c|c|c|}
\hline Parameter & $\begin{array}{l}\text { T2DM, no } \\
\text { HTN*n=101 }\end{array}$ & $\begin{array}{l}\text { HTN, no T2DM** } \\
\mathrm{n}=62\end{array}$ & $\begin{array}{l}\text { T2DM and } \\
\text { HTN }^{* * *} n=76\end{array}$ & $\mathbf{p}$ & $\begin{array}{l}\text { Post hoc multiple } \\
\text { comparisons }\end{array}$ \\
\hline EDD, mm & $51.0 \pm 4.5$ & \begin{tabular}{|l|}
$47.6 \pm 3.4$ \\
\end{tabular} & $49.3 \pm 3.8$ & $<0.001$ & $* \mathrm{vs} * * ; * \mathrm{vs} * * *$ \\
\hline $\mathrm{ESD}, \mathrm{mm}$ & $32.5 \pm 4.5$ & $29.7 \pm 2.9$ & $30.8 \pm 3.1$ & $<0.001$ & $* \mathrm{vs}^{* *} ; * \mathrm{vs}^{* * *}$ \\
\hline Stroke volume, $\mathrm{ml}$ & $78.7 \pm 16.5$ & $71.39 \pm 14.0$ & $77.1 \pm 14.2$ & 0.013 & $* \mathrm{vs}^{* *}$ \\
\hline Ejection fraction, $\%$ & $65.3 \pm 6.4$ & $67.5 \pm 4.7$ & $67.2 \pm 4.7$ & 0.020 & $* \mathrm{vs}^{* *}$ \\
\hline LAVI, $\mathrm{ml} / \mathrm{m}^{2}$ & $25.6 \pm 6.2$ & $25.8 \pm 6.9$ & $24.6 \pm 5.8$ & 0.43 & \\
\hline Left ventricular mass, $g$ & $167.4 \pm 41.6$ & $131.3 \pm 28.3$ & $152.3 \pm 36.1$ & $<0.001$ & $\begin{array}{l}* \mathrm{vs}^{* * ; *} \mathrm{vs}^{* * * *} \\
* * \mathrm{v} \mathrm{v}^{* * * *}\end{array}$ \\
\hline LVMI, $\mathrm{g} / \mathrm{m}^{2}$ & $84.9 \pm 20.3$ & $69.8 \pm 12.4$ & $77.9 \pm 16$ & $<0.001$ & $\begin{array}{l}* \mathrm{vs}^{* * ; *} \mathrm{vs}^{* * * *} \\
* * \mathrm{vs}^{* * * *}\end{array}$ \\
\hline Cardiac index, $1 / \mathrm{min} / \mathrm{m}^{2}$ & $3.1 \pm 0.7$ & $2.8 \pm 0.6$ & $3.2 \pm 0.9$ & 0.06 & \\
\hline $\mathrm{E}, \mathrm{m} / \mathrm{s}$ & $0.61 \pm 0.15$ & $0.63 \pm 0.14$ & $0.62 \pm 0.15$ & 0.65 & \\
\hline $\mathrm{A}, \mathrm{m} / \mathrm{s}$ & $0.68 \pm 0.16$ & $0.69 \pm 0.13$ & $0.76 \pm 0.17$ & 0.002 & $* \mathrm{vs} * * * ; * * \mathrm{vs} * * *$ \\
\hline $\mathrm{E} / \mathrm{A}$ & $\begin{array}{l}0.85 \\
(0.71-1.15)\end{array}$ & $0.88(0.7-1.14)$ & $0.76(0.69-0.92)$ & 0.039 & $* \mathrm{vs} * * *$ \\
\hline $\mathrm{DT}, \mathrm{msec}$ & $216.3 \pm 47.9$ & $228.4 \pm 39.1$ & $233.8 \pm 47.0$ & 0.033 & $* \mathrm{vs} * * *$ \\
\hline E/E' mean & $5.56 \pm 1.7$ & $5.65 \pm 1.39$ & $5.56 \pm 2.0$ & 0.94 & \\
\hline Relative wall thickness & $0.32 \pm 0.07$ & $0.30 \pm 0.04$ & $0.32 \pm 0.05$ & 0.14 & \\
\hline
\end{tabular}

Table 2. Echocardiographic parameters. ANOVA; Data is presented as means \pm SD, median $[25-75$ th percentile], or percentages; T2DM-type 2 diabetes, HTN-hypertension; EDD-left ventricular end diastolic diameter, ESD-left ventricular end systolic diameter, LAVI-left atrial volume index, LVMI-left ventricular mass index, E-early mitral valve flow velocity, A-late mitral valve flow velocity, E/A-ratio of early to late mitral valve flow velocity, DT-deceleration time, E/E'-ratio of early mitral valve flow velocity to early Tissue Doppler lengthening velocity.

\begin{tabular}{|l|l|l|l|}
\hline Parameter & OR & 95\% Confidence interval & $\mathbf{p}$ \\
\hline Age, years & 1.052 & $0.999-1.109$ & 0.05 \\
\hline Female sex & 0.595 & $0.263-1.345$ & 0.21 \\
\hline Body mass index, $\mathrm{kg} / \mathrm{m}^{2}$ & 1.200 & $1.070-1.347$ & 0.002 \\
\hline Systolic blood pressure, $\mathrm{mmHg}$ & 0.998 & $0.932-1.069$ & 0.96 \\
\hline Diastolic blood pressure, $\mathrm{mmHg}$ & 0.847 & $0.768-0.934$ & 0.001 \\
\hline Ejection fraction, \% & 0.956 & $0.885-1.034$ & 0.26 \\
\hline LVMI, g/m ${ }^{2}$ & 1.033 & $1.003-1.065$ & 0.029 \\
\hline E/A & 0.828 & $0.150-4.554$ & 0.83 \\
\hline DT, msec & 0.994 & $0.985-1.003$ & 0.19 \\
\hline E/E' mean & 0.978 & $0.768-1.244$ & 0.86 \\
\hline
\end{tabular}

Table 3. Multivariable analysis of baseline characteristics and echocardiographic parameters associated with type 2 diabetes. LVMI-left ventricular mass index, E/A-ratio of early to late mitral valve flow velocity, DTdeceleration time, E/E'-ratio of early mitral valve flow velocity to early Tissue Doppler lengthening velocity.

\begin{tabular}{|l|l|l|l|l|}
\hline Parameter & $\boldsymbol{\beta}$ coefficient & SE & $\boldsymbol{\beta}$ standarized & $\mathbf{p}$ \\
\hline Type 2 diabetes & 5.898 & 2.806 & 0.143 & 0.037 \\
\hline Female sex & -9.200 & 2.184 & -0.254 & $<0.001$ \\
\hline E/E' mean & 2.814 & 0.617 & 0.268 & $<0.001$ \\
\hline Hypertension & -6.552 & 2.491 & -0.179 & 0.009 \\
\hline E/A & -9.604 & 3.960 & -0.143 & 0.016 \\
\hline
\end{tabular}

Table 4. Multivariable analysis of baseline characteristics and echocardiographic parameters associated with left ventricular mass index. LVMI-left ventricular mass index, E/A-ratio of early to late mitral valve flow velocity, $\mathrm{E} / \mathrm{E}^{\prime}$-ratio of early mitral valve flow velocity to early Tissue Doppler lengthening velocity.

of T2DM and HTN lead to the highest LVMI, followed by patients with only HTN and T2DM, respectively ${ }^{25}$. Although a long asymptomatic period may precede T2DM symptoms and diagnosis, and reported duration of the disease may be underestimated, patients included in our study were considered to be at the beginning of cardio-metabolic continuum and early phase of myocardial impairment. Even so, largest LVMI in this group indicates the possible negative effect of hyperglycemia on LV mass increase, even before overt T2DM. Furthermore, there is data supportive of the link between pre-diabetic states (insulin resistance, impaired fasting glucose and impaired glucose tolerance) with increase in LV mass ${ }^{25-27}$. Also, worse glycemic control reflected in higher HbA1c 


\begin{tabular}{|l|l|l|l|}
\hline & Parameter & r & p \\
\hline & Female sex & -0.2721 & $<0.001$ \\
\cline { 2 - 4 } & Glucose, mmol/l & 0.2652 & $<0.001$ \\
\cline { 2 - 4 } & Glycated hemoglobin, \% & 0.2311 & 0.0003 \\
\cline { 2 - 4 } Left ventricular mass index, $\mathrm{g} / \mathrm{m}^{2}$ & HDL cholesterol, $\mathrm{mmol} / \mathrm{l}$ & -0.2089 & 0.001 \\
\cline { 2 - 4 } & Creatinine, $\mu \mathrm{mmol} / \mathrm{l}$ & 0.3186 & $<0.001$ \\
\cline { 2 - 4 } & eGFR, $\mathrm{ml} / \mathrm{min} / 1.73 \mathrm{~m}^{2}$ & -0.1410 & 0.030 \\
\cline { 2 - 4 } & Systolic blood pressure, $\mathrm{mmHg}$ & -0.2281 & 0.0004 \\
\cline { 2 - 4 } & Diastolic blood pressure, $\mathrm{mmHg}$ & -0.2408 & 0.0002 \\
\hline
\end{tabular}

Table 5. Correlations of left ventricular mass index and baseline characteristics. r-Pearson's correlation coefficient; HDL-high density lipoprotein; eGFR-estimated glomerular filtration rate.

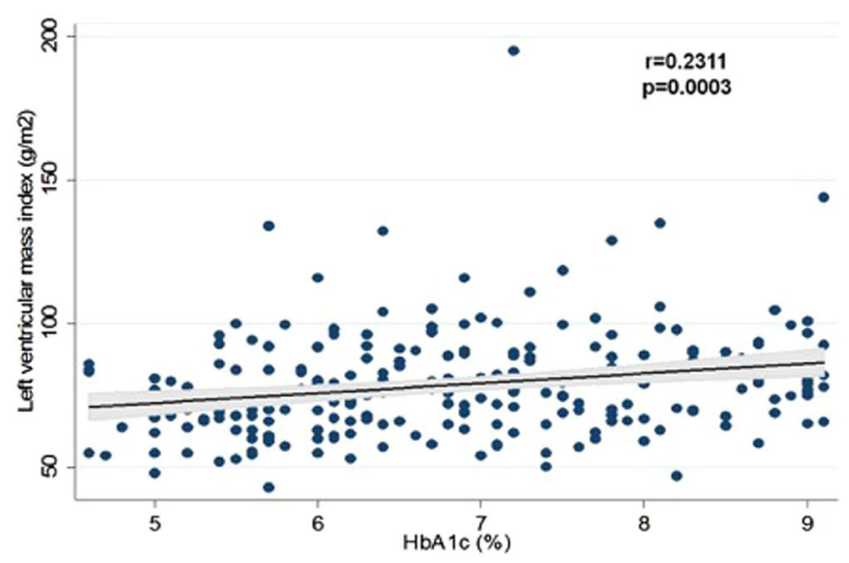

Figure 1. Correlation of left ventricular mass index and glucose.

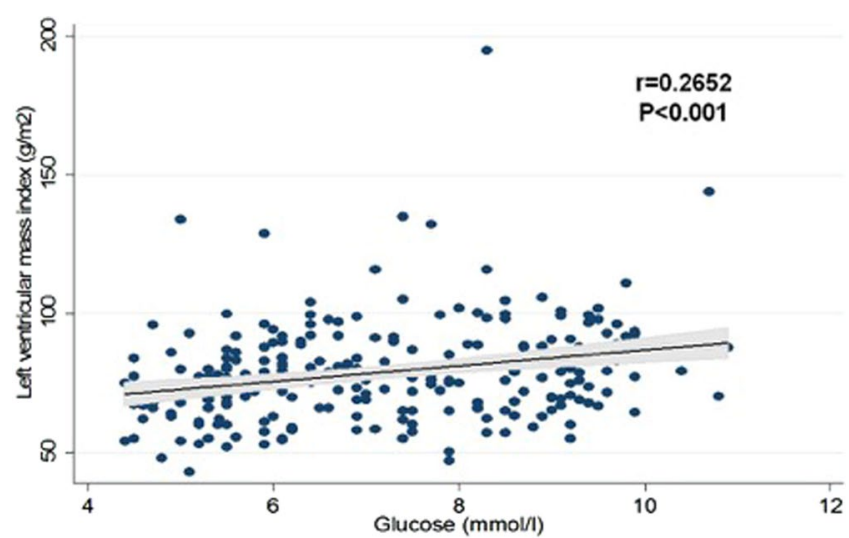

Figure 2. Correlation of left ventricular mass index and HbA1c.

has been associated with LV mass increase ${ }^{28,29}$, which is in line with our results. In a large community-based cohort, participants with T2DM had a steeper increase in LV mass over time compared to those without T2DM ${ }^{30}$.

It has been suggested that T2DM induces LV mass enlargement through "metabolic", and not hemodynamic pathways ${ }^{31,32}$. Hyperglycaemia and hyperinsulinemia cause interstitial deposition of advanced-glycated end products, increased serum aldosterone levels causing myocyte growth and changes in the extracellular matrix, and activation of cytokines and angiotensin II, all leading to myocardial fibrosis and subsequent increase in LV mass $^{33,34}$. Hence, LVMI increase seems to be present in T2DM despite no increase in afterload, suggesting the existence of an isolated effect of T2DM on the structural remodeling of the heart. In the Strong Heart Study, early cardiac impairment in T2DM was characterized by increased LV mass and subclinical LV dysfunction ${ }^{35}$. In our T2DM cohort, the only signal of cardiac impairment was increased LVMI, while diastolic and systolic function were normal. We therefore speculate that increase in LV mass could be an early marker of cardiac dysfunction in T2DM patients, developing even prior to the asymptomatic diastolic dysfunction. Timely identifying increase in LV mass is very important, as it has been shown to be a strong predictor of sudden cardiac death ${ }^{36,37}, \mathrm{CV}$ disease $^{38}$, 
and all-cause mortality in both middle-aged ${ }^{39}$, and elderly individuals ${ }^{40}$. A similar notion has been proposed by Levy et al., who suggested that LV mass provides additional prognostic information to the traditional CV risk factors in the general population ${ }^{41}$. Therefore, the results of the current study uphold the European Society of Cardiology and European Association for the Study of Diabetes Guidelines on diabetes, pre-diabetes, and cardiovascular diseases ${ }^{42}$ which suggest that echocardiography should be considered as a regular screening tool in all T2DM patients, even if they are asymptomatic and without overt cardiovascular disease $\mathrm{e}^{43,44}$. This interesting finding points out the importance of identifying patients at high risk for $\mathrm{CV}$ events and also sets the stage for the future investigation which should determine the role of LVMI as a potential independent CV risk factor.

Several limitations of current study need to be mentioned. This analysis was cross-sectional, and therefore no conclusions on causality could be drawn. Coronary angiography was not performed, due to the invasiveness and cost, hence all patients underwent stress echocardiography, which is considered to have the sensitivity of $85 \%$ and specificity of $77 \%$ for the detection of coronary artery disease ${ }^{45}$. Glucose clamp, the gold standard in the assessment of insulin sensitivity was not used in this study. As HOMA-IR was previously shown to strongly correlate with clamp-measured insulin resistance, we used this reliable and applicable diagnostic tool.

\section{Conclusions}

LVMI was largest in patients with only T2DM, which suggests that it could be a potential, pre-symptomatic marker of myocardial structural change in T2DM. Also, LVMI was associated with higher fasting glucose and $\mathrm{HbAlc}$, indicating the possible role of hyperglycemia in LV mass increase.

\section{References}

1. Huxley, R., Barzi, F. \& Woodward, M. Excess risk of fatal coronary heart disease associated with diabetes in men and women: metaanalysis of 37 prospective cohort studies. BMJ. 332(7533), 73-8 (2006).

2. Kannel, W. B., Hjortland, M. \& Castelli, W. P. Role of diabetes in congestive heart failure: the Framingham study. Am J Cardiol. 34, 29-34 (1974).

3. Pocock, S. J. et al. Predictors of mortality and morbidity in patients with chronic heart failure. Eur Heart J. 27, 65-75 (2006).

4. Skali, H. et al. Cardiac structure and function across the glycemic spectrum in elderly men and women free of prevalent heart disease: the Atherosclerosis Risk In the Community study. Circ Heart Fail. 8(3), 448-54 (2015).

5. Heckbert, S. R. et al. Traditional cardiovascular risk factors in relation to left ventricular mass volume and systolic function by cardiac magnetic resonance imaging: the Multiethnic Study of Atherosclerosis. J Am Coll Cardiol. 48, 2285-92 (2006).

6. Eguchi, K. et al. Association between diabetes mellitus and left ventricular hypertrophy in a multiethnic population. Am J Cardiol. 101(12), 1787-91 (2008).

7. Fonseca, C. G. et al. Three-dimensional assessment of left ventricular systolic strain in patients with type 2 diabetes mellitus, diastolic dysfunction, and normal ejection fraction. Am J Cardiol. 94(11), 1391-5 (2004).

8. De Simone, G., Pasanisi, F. \& Contaldo, F. Link of non-hemodynamic factors to hemodynamic determinants of left ventricular hypertrophy. Hypertension. 38, 13-8 (2001).

9. De Simone, G., Verdecchia, P., Pede, S., Gorini, M. \& Maggioni, A. P. Prognosis of inappropriate left ventricular mass in hypertension: the MAVI Study. Hypertension. 40, 470-6 (2002).

10. Kuperstein, R., Hanly, P., Niroumand, M. \& Sasson, Z. The importance of age and obesity on the relation between diabetes and left ventricular mass. J Am Coll Cardiol. 37, 1957-62 (2001).

11. Jørgensen, P. G. et al. Abnormal echocardiography in patients with type 2 diabetes and relation to symptoms and clinical characteristics. Diab Vasc Dis Res. 13(5), 321-30 (2016).

12. Redfield, M. M. et al. Burden of systolic and diastolic ventricular dysfunction in the community: appreciating the scope of the heart failure epidemic. JAMA. 289, 194-202 (2003).

13. Srivastava, P. M. et al. Prevalence and predictors of cardiac hypertrophy and dysfunction in patients with Type 2 diabetes. Clin Sci. 114, 313-20 (2008).

14. Ichikawa, R. et al. Influencing factors on cardiac structure and function beyond glycemic control in patients with type 2 diabetes mellitus. Cardiovasc Diabetol. 12, 38 (2013).

15. James, P. A. et al. 2014 evidence-based guideline for the management of high blood pressure in adults: report from the panel members appointed to the Eighth Joint National Committee (JNC 8). JAMA. 311(5), 507-20 (2014).

16. Levey, A. S. et al. A more accurate method to estimate glomerular filtration rate from serum creatinine: a new prediction equation. Modification of diet in renal disease study group. Ann Intern Med. 130, 461-70 (1999).

17. Matthews, D. R. et al. Homeostasis model assessment: insulin resistance and beta-cell function from fasting plasma glucose and insulin concentrations in man. Diabetologia. 28, 412-9 (1985).

18. Bruce, R. A. Exercise testing of patients with coronary heart disease. Ann Clin Res. 3, 323-30 (1971).

19. Lang, R. M. et al. Recommendations for cardiac chamber quantification by echocardiography in adults: an update from the American Society of Echocardiography and the European Association of Cardiovascular Imaging. Eur Heart J Cardiovasc Imaging. 16(3), 233-70 (2015).

20. Devereux, R. B. et al. Echocardiographic assessment of left ventricular hypertrophy: comparison to necropsy findings. Am J Cardiol. 57, 450-8 (1986).

21. Marwick, T. H. et al. Recommendations on the Use of Echocardiography in Adult Hypertension: A Report from the European Association of Cardiovascular Imaging (EACVI) and the American Society of Echocardiography (ASE). J Am Soc Echocardiogr. 28(7), 727-54 (2015).

22. de Simone, G. et al. Prognostic implications of the compensatory nature of left ventricular mass in arterial hypertension. J Hypertens. 19, 119-25 (2001).

23. Tesic, M. et al. N-terminal pro-brain natriuretic peptide is related with coronary flow velocity reserve and diastolic dysfunction in patients with asymmetric hypertrophic cardiomyopathy. J Cardiol. 70(4), 323-328 (2017).

24. Levelt, E. et al. Relationship Between Left Ventricular Structural and Metabolic Remodeling in Type 2 Diabetes. Diabetes. 65(1), 44-52 (2016).

25. Liu, J. E. et al. The impact of diabetes on left ventricular filling pattern in normotensive and hypertensive adults: the Strong Heart Study. J Am Coll Cardiol. 37(7), 1943-9 (2001).

26. Velagaleti, R. S. et al. Relations of insulin resistance and glycemic abnormalities to cardiovascular magnetic resonance measures of cardiac structure and function: the Framingham Heart Study. Circ Cardiovasc Imaging. 3, 257-63 (2010).

27. Coats, A. J. \& Anker, S. D. Insulin resistance in chronic heart failure. J Cardiovasc Pharmacol. 35(Suppl 4), S9-14 (2000). 7.

28. Shah, R. V. et al. Insulin resistance, subclinical left ventricular remodeling, and the obesity paradox: MESA (Multi-Ethnic Study of Atherosclerosis). J Am Coll Cardiol. 61, 1698-706 (2013). 
29. Sesti, G. et al. Characterization of left ventricular mass in individuals at risk for type 2 diabetes identified by HbAlc levels according to the American Diabetes Association criteria. Int J Cardiol. 179, 211-3 (2015).

30. Lieb, W. et al. Longitudinal tracking of left ventricular mass over the adult life course: clinical correlates of short- and long-term change in the Framingham Offspring Study. Circulation. 119(24), 3085-92 (2009).

31. Cheng, S. et al. Correlates of echocardiographic indices of cardiac remodeling over the adult life course longitudinal observations from the Framingham Heart Study. Circulation. 122, 570-8 (2010).

32. Joshi, D. et al. Continuous, noninvasive measurement of the haemodynamic response to submaximal exercise in patients with diabetes mellitus: evidence of impaired cardiac reserve and peripheral vascular response. Heart. 96, 36-41 (2010).

33. Scheuermann-Freestone, M. et al. Abnormal cardiac and skeletal muscle energy metabolism in patients with type 2 diabetes. Circulation. 107, 3040-6 (2003).

34. Larghat, A. M. et al. The microvascular effects of insulin resistance and diabetes on cardiac structure, function, and perfusion: a cardiovascular magnetic resonance study. Eur Heart J Cardiovasc Imaging. 15(12), 1368-76 (2014).

35. Van Hoeven, K. V. \& Factor, S. M. A comparison of the pathological spectrum of hypertensive, diabetic, and hypertensive-diabetic heart disease. Circulation. 82, 848-55 (1990).

36. Devereux, R. B. et al. Impact of diabetes on cardiac structure and function. The Strong Heart Study. Circulation. 101, 2271-6 (2000).

37. Haider, A. W., Larson, M. G., Benjamin, E. J. \& Levy, D. Increased left ventricular mass and hypertrophy are associated with increased risk for sudden death. J Am Coll Cardiol. 32, 1454-9 (1998).

38. Cioffi, G. et al. Inappropriate left ventricular mass independently predicts cardiovascular mortality in patients with type 2 diabetes. Int J Cardiol. 168(5), 4953-6 (2013).

39. Casale, P. N. et al. Value of echocardiographic measurement of left ventricular mass in predicting cardiovascular morbid events in hypertensive men. Ann Intern Med. 105, 173-8 (1986).

40. Levy, D., Garrison, R. J., Savage, D. D., Kannel, W. B. \& Castelli, W. P. Prognostic implications of echocardiographically determined left ventricular mass in the Framingham Heart Study. N Engl J Med. 322, 1561-6 (1990).

41. Gardin, J. M. et al. M-mode echocardiographic predictors of six- to seven year incidence of coronary heart disease, stroke, congestive heart failure, and mortality in an elderly cohort (the Cardiovascular Health Study). Am J Cardiol. 87, 1051-7 (2001).

42. Rydén, L. et al. ESC Guidelines on diabetes, pre-diabetes, and cardiovascular diseases developed in collaboration with the EASD. Eur Heart J. 34, 3035-87 (2013).

43. LJM, B.-deW. et al. High prevalence of previously unknown heart failure and left ventricular dysfunction in patients with type 2 diabetes. Diabetologia. 55, 2154-62 (2012).

44. Young, L. H. et al. DIAD Investigators. Cardiac outcomes after screening for asymptomatic coronary artery disease in patients with type 2 diabetes: the DIAD study: a randomized controlled trial. JAMA. 301, 1547-55 (2009).

45. Fleischmann, K. E., Hunink, M. G., Kuntz, K. M. \& Douglas, P. S. Exercise echocardiography or exercise SPECT imaging? A metaanalysis of diagnostic test performance. JAMA. 280(10), 913-20 (1998).

\section{Author Contributions}

J.P.S., M.T., P.M.S. and N.M.L. designed the study, analyzed data and contributed to discussions on the results. J.P.S., M.T., P.M.S., K.L., A.J., T.B.S., L.L., T.M., M.M., J.S.G. and N.M.L were involved in recruiting patients, wrote the manuscript and were involved in the revision of the manuscript. M.T. did all the echocardiographic measurements. V.G. performed all stress echocardiography tests. S.S. was involved in collecting blood samples and performing biochemical analysis. J.P.S. and N.M. performed statistical analysis. All authors approved the decision to submit the article for publication. edited the manuscript. J.P.S. is a guarantor of the work, and as such, had full access to all the data in the study and takes responsibility for the integrity of the data and the accuracy of the data analysis.

\section{Additional Information}

Supplementary information accompanies this paper at https://doi.org/10.1038/s41598-018-19229-w.

Competing Interests: The authors declare that they have no competing interests.

Publisher's note: Springer Nature remains neutral with regard to jurisdictional claims in published maps and institutional affiliations.

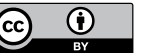

Open Access This article is licensed under a Creative Commons Attribution 4.0 International License, which permits use, sharing, adaptation, distribution and reproduction in any medium or format, as long as you give appropriate credit to the original author(s) and the source, provide a link to the Creative Commons license, and indicate if changes were made. The images or other third party material in this article are included in the article's Creative Commons license, unless indicated otherwise in a credit line to the material. If material is not included in the article's Creative Commons license and your intended use is not permitted by statutory regulation or exceeds the permitted use, you will need to obtain permission directly from the copyright holder. To view a copy of this license, visit http://creativecommons.org/licenses/by/4.0/.

(C) The Author(s) 2018 